\title{
Beneficial effects of intravenous dexmedetomidine on cognitive function and cerebral injury following a carotid endarterectomy
}

\author{
YA-LI GE ${ }^{1}$, XIAOBO LI $^{2}$, JU GAO $^{1}$, XICHENG ZHANG $^{3}$, XIANGZHI FANG ${ }^{1}$, \\ LUOJING ZHOU ${ }^{4}$, WEI JI ${ }^{1}$ and SHUNYAN LIN ${ }^{1}$ \\ Departments of ${ }^{1}$ Anesthesiology, ${ }^{2}$ Neurology, ${ }^{3}$ Vascular Surgery and ${ }^{4}$ Scientific Research, \\ Subei People's Hospital of Jiangsu Province, Yangzhou, Jiangsu 225001, P.R. China
}

Received May 11, 2015; Accepted November 25, 2015

DOI: $10.3892 /$ etm.2016.2978

\begin{abstract}
The present study aimed to investigate the effects of dexmedetomidine (DEX) on cognition following a carotid endarterectomy (CEA). In addition, the neuroprotective effects of DEX against ischemia-reperfusion injury during CEA were analyzed. Patients due to undergo elective CEA under general anesthesia were randomly assigned to either the DEX-treated group (group $\mathrm{D} ; \mathrm{n}=25$ ) or the control group (group $\mathrm{C} ; \mathrm{n}=25$ ). Patients in group D were treated with $0.3 \mu \mathrm{g} / \mathrm{kg}$ DEX pre-CEA, followed by $0.3 \mu \mathrm{g} / \mathrm{kg} / \mathrm{h}$ DEX intraoperatively up to $30 \mathrm{~min}$ prior to the completion of surgery, and the patients in group $\mathrm{C}$ received an equal volume of normal saline. Cognitive function was assessed prior to CEA (T0), and at 24,48 , and $72 \mathrm{~h}$, 7 days and 1 month post-surgery (T1-5, respectively), using the Mini-Mental State Examination (MMSE). Blood samples were drawn from the ipsilateral jugular bulb of all patients at $20 \mathrm{~min}$ prior to anesthesia ( $\mathrm{t} 0$ ), and at $10 \mathrm{~min}$ following tracheal intubation, $15 \mathrm{~min}$ following clamping and unclamping of the carotid artery, and at 6 and $24 \mathrm{~h}$ postoperatively (t1-5, respectively). The protein expression levels of markers of cerebral ischemia and injury, namely S100 calcium-binding protein B (S100B) and neuron-specific enolase (NSE), and the concentration of the oxidative stress marker malondialdehyde (MDA), were analyzed. Patients in group D exhibited elevated MMSE scores at T2 and T3 post-CEA, as compared with group C. Furthermore, the protein expression level of S100B and the concentration of MDA in the jugular bulb of group D patients were markedly decreased compared with those in group $\mathrm{C}$ at $\mathrm{t} 3-5$ and $\mathrm{t} 3$, respectively. The results of the present study suggested that DEX was able to enhance the recovery
\end{abstract}

Correspondence to: Dr Ju Gao, Department of Anesthesiology, Subei People's Hospital of Jiangsu Province, 98 Nan Tong Western Road, Yangzhou, Jiangsu 225001, P.R. China

E-mail: happygezi81@163.com

Key words: carotid endarterectomy, dexmedetomidine, cognitive function, Mini-Mental State Examination, S100 calcium-binding protein B, malondialdehyde of cognition following CEA, and this was associated with decreased cerebral damage and antioxidative effects.

\section{Introduction}

A carotid endarterectomy (CEA) is a surgical procedure that is performed in order to remove deposits of fat, called plaque, from the carotid arteries in the neck (1). Plaque builds up in large- and medium-sized arteries as people get older, and this build up is a vascular disease called atherosclerosis (1). During a CEA, a surgeon removes the fatty deposits in order to correct the narrowing and allow blood and oxygen to flow freely to the brain (1). Previous studies have demonstrated that conducting a CEA in patients with significant carotid stenosis, including those with or without symptoms, is successful in preventing stroke in $>70 \%$ of cases $(2,3)$. However, the effects of CEA on cognitive function remain unclear (4,5). Irvine et al (6) reported that cognitive improvements were detected in $15 / 22$ studies that had previously investigated patients undergoing CEA. Conversely, other reports have suggested that the CEA procedure may exert limited effects on cognitive function, and subtle cognitive dysfunction has been detected in 24-28\% of patients following CEA $(7,8)$, which may have been associated with alterations in the distribution of cerebral flow, intraoperative cerebral ischemia and reperfusion injury.

Dexmedetomidine (DEX), which is the dextro-enantiomer of medetomidine, is a highly selective $\alpha 2$-adrenoreceptor $(\alpha 2-\mathrm{AR})$ agonist. It has been shown to exhibit various properties, including sedative, analgesic and sympatholytic activities, and an ability to stabilize hemodynamics. DEX is considered a useful adjuvant to general anesthesia, since it is able to decrease the required doses of anesthetics and analgesics, and promote hemodynamic stability (9). In addition, previous studies have demonstrated neuroprotective effects for DEX in animal models of stroke $(10,11)$. Furthermore, $\alpha 2$-ARs have been found to be closely associated with cognitive function (12), which may be affected by increased activity in the dorsal noradrenergic bundles, where $\alpha 2-\mathrm{AR}$ are located, and in the frontal lobe, where $\alpha 2$-ARs mediate increased attention (13). Therefore, the present study hypothesized that DEX treatment may improve cognitive performance in patients following CEA.

The present study aimed to investigate the effects of the intravenous administration of DEX, as an adjunct to general 
anesthesia, on cognitive function and cerebral injury in patients post-CEA. In addition, the protein expression levels of the cerebral ischemia and injury markers S100 calcium-binding protein B (S100B) and neuron-specific enolase (NSE), and the concentration of the oxidative stress marker malondialdehyde (MDA), were determined.

\section{Materials and methods}

Ethics statement and patients. The present study was approved by the Bioethics Committee at the Subei People's Hospital of Jiangsu Province (no. 2011032). The exclusion criteria were as follows: i) The patient refused general anesthetic or enrollment into the present study, ii) the patient was undergoing treatment with psychiatric drugs, iii) the patient had an initial Mini-Mental State Examination (MMSE) score of $<20$, iv) the patient was unable to write or spell, and v) the patient underwent emergency surgery. Patients included in the present study were diagnosed with a carotid stenosis of $\geq 70 \%$, with or without symptoms, and had not previously undergone carotid surgery. Written informed consent was obtained from all patients. A total of 50 patients due to undergo elective CEA were randomized, using computer-generated random numbers, into two groups: The study group (group $\mathrm{D} ; \mathrm{n}=25$ ) and the control group (group $\mathrm{C} ; \mathrm{n}=25$ ). The mean age of the patients in group $\mathrm{C}$ was $72 \pm 4$ years (age range, 67-83 years) and the male/female ratio was $13 / 11$. The mean age of the patients in group $\mathrm{D}$ was $70 \pm 3$ years (age range, $65-84$ years) and the male/female ratio was $16 / 8$. Reasons to abandon the protocol included the use of anesthetic drugs other than those indicated in the protocol, the use of an intraoperative shunt, diagnosis of severe hypotension, administration of a second anesthetic within the first $24 \mathrm{~h}$, and an inability to perform a MMSE evaluation.

Anesthetic management. On the evening prior to surgery, patients were orally administered $25 \mathrm{mg}$ clorazepate (Wanbang Biopharmaceuticals, Co., Ltd., Xuzhou, China) in order to decrease the preoperative anxiety of the patients; however, no oral pre-medication was administered on the day of surgery. Anesthesia was induced with intravenous midazolam (0.05-0.1 mg/kg; Enhua Pharmaceutical Group, Co., Ltd., Xuzhou, China), fentanyl ( $2 \mu \mathrm{g} / \mathrm{kg}$; Renfu Nuosheng Pharmaceutical, Co., Ltd., Wuhan, China) and etomidate (0.2-0.3 mg/kg; Enhua Pharmaceutical Group, Co., Ltd.). Following treatment with rocuronium bromide $(1.0 \mathrm{mg} / \mathrm{kg}$; Xincheng Company, Lianyungang, China), the trachea was intubated using an armored endotracheal tube (Meinuo Medical Appliances, Co., Ltd., Suzhou, China). In order to maintain a normal end-tidal $\mathrm{CO}_{2}\left(\mathrm{ETCO}_{2}, 30-40 \mathrm{mmHg}\right)$, the mechanical ventilation parameters were set as follows: Tidal volume, $8 \mathrm{ml} / \mathrm{kg}$; fraction of inspired $\mathrm{O}_{2}\left(\mathrm{FiO}_{2}\right), 0.5$; and breathing frequency, $10-14$ breaths/min. Anesthesia was maintained with the intravenous infusion of remifentanil (0.1-0.3 $\mu \mathrm{g} / \mathrm{kg} / \mathrm{min}$; Renfu Nuosheng Pharmaceutical, Co., Ltd.) and propofol (2-6 mg/kg/h); the infusion rate was adjusted according to the bispectral index. In addition, patients were intermittently administered rocuronium bromide $(0.1 \mathrm{mg} / \mathrm{kg})$, in order to maintain muscle relaxation.

Group D patients were intravenously administered $0.3 \mu \mathrm{g} / \mathrm{kg}$ DEX 10 min prior to anesthesia, and then at a rate of $0.3 \mu \mathrm{g} / \mathrm{kg} / \mathrm{h}$ intraoperatively until $30 \mathrm{~min}$ prior to the completion of surgery. The patients in group $\mathrm{C}$ received an equal volume of normal saline. The mean arterial blood pressure (MAP) was controlled intraoperatively within $\pm 20 \%$ of the baseline value. During clamping of the carotid artery, MAP values were maintained at $+25 \%$ of the baseline by treating the patients with dopamine (2-8 $\mu \mathrm{g} / \mathrm{kg} / \mathrm{min})$ or nitroglycerine $(0.1-8.0 \mu \mathrm{g} / \mathrm{kg} / \mathrm{min})$, as appropriate. Following unclamping of the carotid artery, the surgical field was infiltrated with $10 \mathrm{ml}$ ropivacaine $(5 \mathrm{mg} / \mathrm{ml}$; Hengrui Medicine, Co., Ltd., Lianyungang, China) for analgesia. All patients were treated with tropisetron $(4 \mathrm{mg} / \mathrm{kg}$; Jiangsu YANGZIJIANG Pharmaceutical Group, Co., Ltd. Taizhou, China) intraoperatively to prevent postoperative nausea and vomiting. The anesthetists were not blinded to the study protocol; however, the surgeons and post anesthetic care unit staff were blinded.

Monitoring indicators. Intraoperative monitoring was conducted using the Philips IntelliVue MP50 patient monitor (Philips Healthcare, Hamburg, Germany), which incorporates a five-lead electrocardiogram, and is able to assess invasive arterial blood pressures, pulse oximetry and $\mathrm{ETCO}_{2}$. The depth of sedation was measured using the A-2000 ${ }^{\mathrm{TM}}$ XP bispectral index monitor (Aspect Medical Systems, Inc., Norwood, MA, USA). MAPs were recorded at $20 \mathrm{~min}$ pre-anesthesia, immediately and at $10 \mathrm{~min}$ following tracheal intubation, at 5, 10 and 15 min following clamping of the carotid artery, and at 5 and 10 min following unclamping of the carotid artery. A shunt was inserted and the patient was excluded from the present study if the carotid artery stump pressure was $<50 \mathrm{mmHg}$ following clamping. One peripheral venous catheter and a radial arterial line for continuous monitoring of arterial blood pressure were inserted. A retrograde internal jugular vein catheter, ipsilateral to the operated carotid, was inserted into the jugular bulb, in order to obtain blood samples. Blood samples were drawn from the jugular bulb $20 \mathrm{~min}$ prior to anesthesia (t0), $10 \mathrm{~min}$ following tracheal intubation (t1), 15 min following clamping of the carotid artery (t2), 15 min following unclamping of the carotid artery (t3), and at 6 and $24 \mathrm{~h}(\mathrm{t} 4-5)$ post-CEA. The blood samples $(2 \mathrm{ml})$ were centrifuged at 2,054 $\mathrm{x}$ g for $15 \mathrm{~min}$ at room temperature, and the serum was maintained at $-80^{\circ} \mathrm{C}$ until further analysis.

Quantification of the serum protein expression levels of $S 100 B$ and NSE, and the plasma concentration of MDA. The serum protein expression levels of S100B and NSE were measured using S100B and NSE enzyme-linked immunosorbent assay kits (cat nos. MY1168 and MY1163, respectively; Nanjing Jiancheng Bioengineering Institute, Nanjing, China), according to the manufacturer's protocol. Plasma MDA levels were determined using high performance liquid chromatography (HPLC; Shimadzu Corporation, Kyoto, Japan), as demonstrated in a previous study (14), although with minor alterations. Briefly, $10 \mu \mathrm{l}$ blood samples were mixed with $190 \mu \mathrm{l} \mathrm{NaOH}(1.3 \mathrm{M})$, and were incubated at $60^{\circ} \mathrm{C}$ for $60 \mathrm{~min}$. Subsequently, $100 \mu \mathrm{l}$ $35 \% \mathrm{HClO}_{4}$ was added to the cooled mixture, and centrifuged at $10,000 \mathrm{x} \mathrm{g}$ for $10 \mathrm{~min}$ at $4^{\circ} \mathrm{C}$. The supernatants of the samples $(300 \mu \mathrm{l})$ were transferred into $1.5 \mathrm{ml}$ HPLC tubes, after which $50 \mu \mathrm{l} 5 \mathrm{mM}, 2$,4-dinitrophenylhydrazine solution was added to the mixture and incubated for $30 \mathrm{~min}$ at room 
Table I. Patient demographics and other clinical data.

\begin{tabular}{|c|c|c|c|}
\hline Variable & Group C & Group D & P-value \\
\hline Age (years) & $72 \pm 4$ & $70 \pm 3$ & 0.06 \\
\hline \multicolumn{4}{|l|}{ Gender } \\
\hline Male & $13(54.2)$ & $16(66.7)$ & \multirow[t]{2}{*}{0.37} \\
\hline Female & $11(45.8)$ & $8(33.3)$ & \\
\hline BMI $\left(\mathrm{kg} / \mathrm{m}^{2}\right)$ & $27 \pm 3$ & $27 \pm 4$ & 0.72 \\
\hline \multicolumn{4}{|l|}{ ASA grade } \\
\hline I & $0(0)$ & $0(0)$ & \multirow[t]{3}{*}{0.23} \\
\hline II & $17(70.8)$ & $13(54.2)$ & \\
\hline III & $7(29.2)$ & $11(45.8)$ & \\
\hline Lateral carotid stenosis $\geq 70 \%$ & $2(8.3)$ & $4(16.7)$ & 0.38 \\
\hline Peripheral artery disease & $2(8.3)$ & $3(12.5)$ & 0.64 \\
\hline Coronary artery disease & $8(33.3)$ & $9(37.5)$ & 0.76 \\
\hline Hypertension & $21(87.5)$ & $22(91.7)$ & 0.63 \\
\hline Diabetes mellitus & $8(33.3)$ & $5(20.8)$ & 0.32 \\
\hline Hyperlipidemia & $13(54.2)$ & $17(70.8)$ & 0.23 \\
\hline Smoker & $12(50.0)$ & $8(33.3)$ & 0.24 \\
\hline Antiplatelet drugs & $16(66.7)$ & $14(58.3)$ & 0.55 \\
\hline Calcium channel blockers & $7(29.2)$ & $10(41.7)$ & 0.37 \\
\hline Statins & $13(54.2)$ & $15(62.5)$ & 0.56 \\
\hline Usage of vasoconstrictors & $11(45.8)$ & $15(62.5)$ & 0.25 \\
\hline Usage of nitroglycerine & $15(62.5)$ & $8(33.3)^{\mathrm{a}}$ & 0.04 \\
\hline Usage of atropine & $3(12.5)$ & $8(33.3)$ & 0.08 \\
\hline Anesthesia duration (min) & $124 \pm 8$ & $120 \pm 5$ & 0.09 \\
\hline Clamp duration (min) & $31 \pm 4$ & $28 \pm 3$ & 0.06 \\
\hline \multicolumn{4}{|l|}{ Endarterectomy site } \\
\hline Left & $15(62.5)$ & $13(54.2)$ & \multirow[t]{2}{*}{0.56} \\
\hline Right & $9(37.5)$ & $11(45.8)$ & \\
\hline Dosage of propofol (mg) & $572 \pm 37$ & $542 \pm 36^{a}$ & 0.01 \\
\hline Postoperative hospitalization (days) & $4.57 \pm 1.12$ & $4.63 \pm 1.30$ & 0.89 \\
\hline
\end{tabular}

Data are presented as the mean \pm standard deviation or $\mathrm{n}(\%)$, and were compared using two-tailed independent $\mathrm{t}$-test or Pearson's $\chi^{2}$ test, respectively ( $n=24$ patients/group). There were no significant differences in demographic parameters between the groups. The usage of nitroglycerine and the dosage of propofol were significantly lower in group $\mathrm{D}$, as compared with group C. ${ }^{\text {P }}<0.05$ vs. group C. BMI, body mass index; ASA, American Society of Anesthesiologists.

temperature. Subsequently, 40- $\mu 1$ samples were injected into the HPLC instrument. The levels of MDA are presented as the concentration of MDA in $\mathrm{nmol} / \mathrm{ml}$.

MMSE scoring. In order to evaluate cognitive function, all patients underwent the MMSE 1 day prior to surgery (T0), and at 24, 48 and $72 \mathrm{~h}$ (T1-3), and 7 days and 1 month post-CEA (T4-5). MMSE tests were conducted by a single psychologist blinded to the study groups. The MMSE scoring system ranges from 0-30, with higher scores indicating a superior cognitive performance.

Sample size analysis. Power analysis was conducted at 1 and $24 \mathrm{~h}$ post-CEA on MMSE scores from 10 patients in a pilot study, in order to assess what may be classed as a significantly different MMSE score between the two groups. The pilot study was designed to achieve a power of $\geq 0.80$ and two-tailed $\alpha$ of 0.05 ; with these parameters, the total required number of participants was 38 . Therefore, a total of 50 participants was deemed an adequate sample size in the present study in order to ensure confidence in the obtained results.

Statistical analysis. Data are presented as the mean \pm standard deviation, and categorical variables are presented as percentages. The normal distribution of values was examined using the Kolmogorov-Smirnov test, and comparisons for normally distributed variables were conducted using an independent t-test between the two groups. Non-normally 
distributed variables were analyzed using the Kruskal-Wallis test. In intragroup analyses, involving comparisons of the same subjects at different time points, normally distributed variables were analyzed using the repeated-measures analysis of variance or the paired t-test, and non-normally distributed variables were compared using the Friedman test or the Wilcoxon signed-rank test. The $\chi^{2}$ test was conducted in order to assess statistical differences in the ratio of variables between the two groups. All statistical analyses were conducted using SPSS software, version 19.0 (IBM SPSS, Armonk, NY, USA). $\mathrm{P}<0.05$ was considered to indicate a statistically significant difference.

\section{Results}

Patients and clinical parameters. A total of 50 patients were enrolled into the present study, of which 48 patients fulfilled the inclusion criteria. Two patients were excluded; 1 patient from group $\mathrm{C}$ and 1 patient from group $\mathrm{D}$ due to the use of a shunt and excessive bleeding, respectively. Patient characteristics and clinical data are presented in Table I. There were no significant differences between the two groups regarding age, gender, American Society of Anesthesiologists (ASA) classification, and existing medications and diseases, including hypertension, coronary artery disease and diabetes. Perioperative data, including anesthesia and clamp durations, and the endarterectomy site, were not significantly different between the two groups. The number of patients requiring treatment with vasoconstrictors, including phenylephrine and/or ephedrine, were 11 (45.8\%) in group C and 15 (62.5\%) in group D; however, this was not significantly different $(\mathrm{P}>0.05)$. Nitroglycerine usage was significantly higher in group C (62.5\%), as compared with group D (33.3\%; P 0.04). Three patients (12.5\%) in group $\mathrm{C}$ were treated with atropine, as compared with 8 patients $(33.3 \%)$ in group D; however this difference was not statistically significant $(\mathrm{P}=0.08)$. The dosage of propofol was significantly lower in group D (542 $\pm 36 \mathrm{mg})$, as compared with group C (572 $\pm 37 \mathrm{mg} ; \mathrm{P}=0.01)$. The postoperative duration of hospitalization was not significantly different between the two groups $(4.57 \pm 1.12$ days in group $\mathrm{C}$ vs. $4.63 \pm 1.30$ days in group $\mathrm{D} ; \mathrm{P}=0.89$ ).

Comparison of MAP. MAP was maintained above the baseline $(+25 \%$ of baseline) in both groups during carotid clamping (Fig. 1), and was significantly increased in group C immediately following tracheal intubation, as compared with the baseline $(\mathrm{P} \leq 0.001)$ and group $\mathrm{D}(\mathrm{P}=0.001)$. The $\mathrm{ETCO}_{2}$ was maintained within the target range $(30-40 \mathrm{mmHg})$ in both groups (Fig. 1).

Comparison of MMSE scores. MMSE scores did not differ significantly between the two groups prior to surgery $(\mathrm{P}>0.05$; Fig. 2). However, MMSE scores were significantly declined in both groups at $\mathrm{T} 1(\mathrm{P} \leq 0.001$ for both), and at $\mathrm{T} 2$ in group $\mathrm{C}$ $(\mathrm{P}=0.001)$, as compared with T0. However, MMSE scores were significantly higher in group D at T2 and T3, as compared with group $\mathrm{C}(\mathrm{P}=0.025$ and $\mathrm{P}=0.03$, respectively). Conversely, MMSE scores did not differ significantly between the two groups at T4 and T5, although MMSE scores were markedly increased at T5 (P>0.05; Fig. 2).

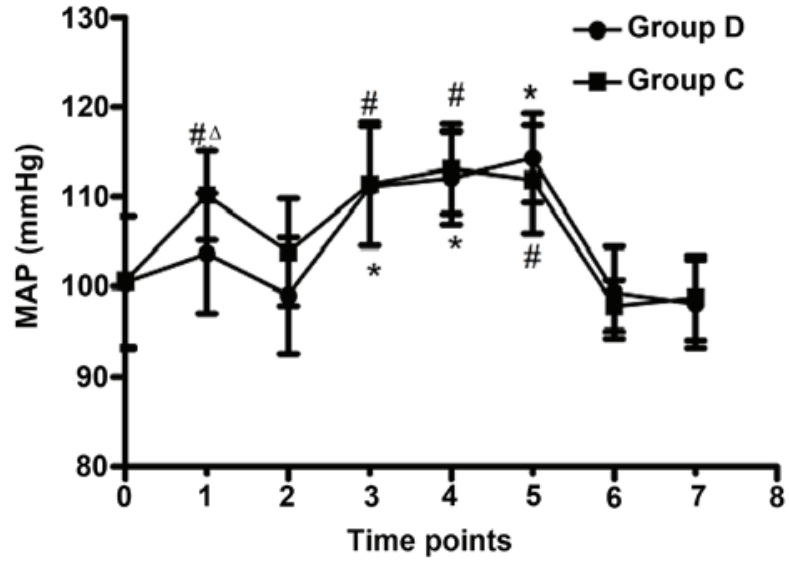

Figure 1. MAP recordings at various time points: 20 min prior to anesthesia (0); immediately following tracheal intubation (1); $10 \mathrm{~min}$ following tracheal intubation (2); 5, 10 and 15 min following clamping of the carotid artery (3-5); and 5 and 10 min following unclamping of the carotid artery $(6,7)$ ${ }^{*} \mathrm{P}<0.05$, vs. the baseline in group $\mathrm{D},{ }^{\#} \mathrm{P}<0.05$ vs. the baseline in group $\mathrm{C}$, ${ }^{\Delta} \mathrm{P}<0.05$ vs. group $\mathrm{D}$ at the same time point in. MAP, mean arterial pressure.

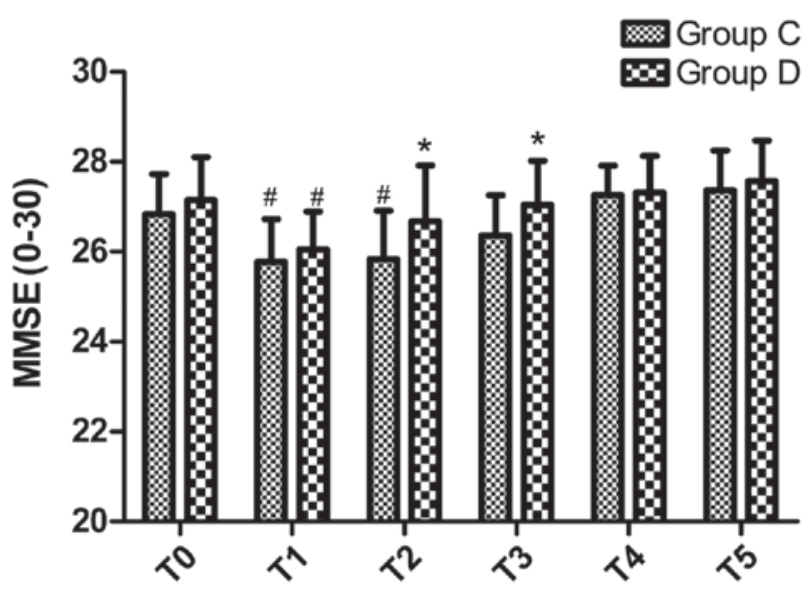

Figure 2. MMSE scores of the two groups on 1 day prior to surgery (T0), and at 24, 48 and $72 \mathrm{~h}$, and 7 days and 1 month post-carotid endarterectomy (T1-5, respectively). ${ }^{*} \mathrm{P}<0.05$ vs. group $\mathrm{C} ;{ }^{\#} \mathrm{P}<0.05$ vs. T0. MMSE, Mini-Mental State Examination score.

Comparison of serum protein expression levels of $S 100 B$ and NSE, and the plasma concentration of MDA. The protein expression levels of S100B and NSE prior to surgery were not significantly different between the two groups $(\mathrm{P}>0.05$; Fig. $3 \mathrm{~A}$ and $\mathrm{B})$. The serum protein expression levels of $\mathrm{S} 100 \mathrm{~B}$ were significantly increased in the two groups at $\mathrm{t} 3-5(\mathrm{P} \leq 0.001)$; however, they were significantly lower in group $\mathrm{D}$ at $\mathrm{t} 3-5$, as compared with group $\mathrm{C}(\mathrm{P}=0.005,0.005$ and 0.001 , respectively; Fig. 3A). There were no significant differences in the serum protein expression levels of NSE between the two groups at any time point (Fig. 3B).

The plasma concentration of MDA increased in the two groups at $\mathrm{t} 3$ and $\mathrm{t} 4$ ( $\mathrm{P} \leq 0.001$ for all); however, it was significantly decreased in group $\mathrm{D}$ at $\mathrm{t} 3$, as compared with group $\mathrm{C}(\mathrm{P}=0.017$; Fig. $3 \mathrm{C})$.

\section{Discussion}

The results of the present study suggested that DEX infusion during CEA may aid the recovery of postoperative cognitive 
A

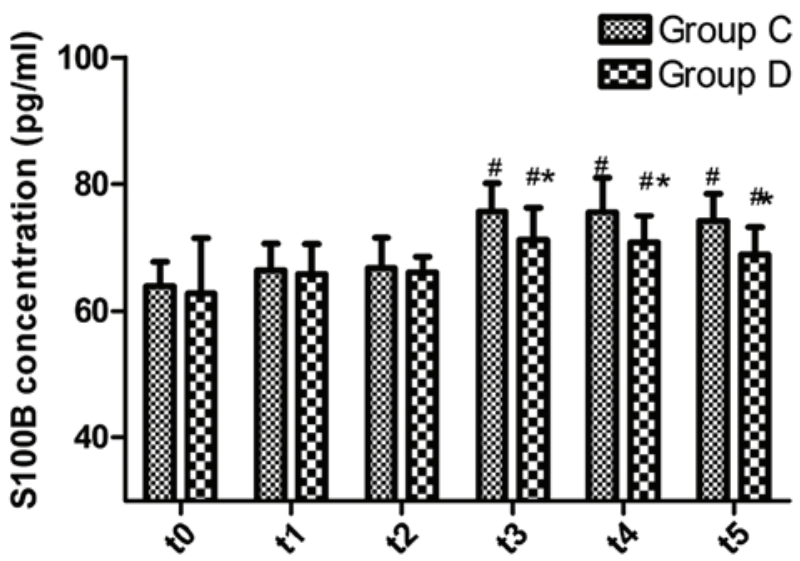

B

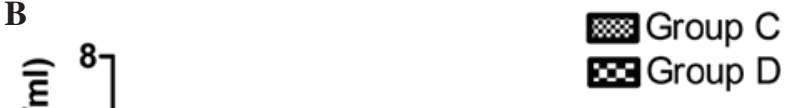

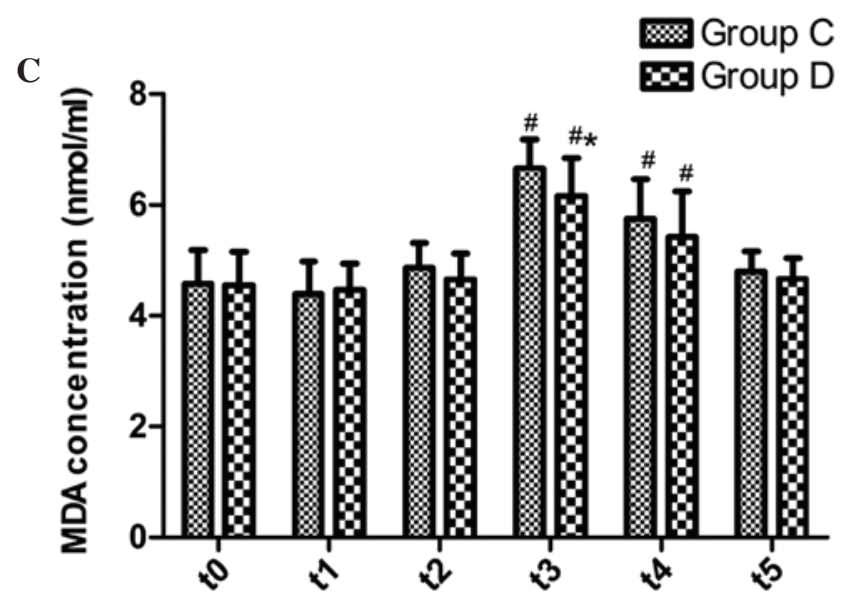

Figure 3. Protein expression levels of (A) S100B and (B) NSE and (C) concentration of MDA in the jugular bulb of group C and group D patients at 20 min prior to anesthesia (t0), 10 min following tracheal intubation (t1), 15 min following clamping of the carotid artery (t2), 15 min following unclamping of the carotid artery (t3), and 6 and $24 \mathrm{~h}$ (t4 and t5) post-carotid endarterectomy. ${ }^{*} \mathrm{P}<0.05$ vs group C; ${ }^{*} \mathrm{P}<0.05 \mathrm{vs}$ t0. S100B, S100 calcium-binding protein $\mathrm{B}$; NSE, neuron-specific enolase; MDA, malondialdehyde.

function, as demonstrated by elevated MMSE scores and increased protein expression levels of S100B and NSE in group D at various time points post-CEA, as compared with group C. In addition, DEX infusion during CEA was associated with decreased concentrations of MDA.

In the present study, the number of patients requiring atropine and vasoconstrictor intervention for the treatment of bradycardia and severe hypotension, respectively, was comparable between the two groups. These results suggested that intravenously administered DEX at the dose used in the present study may be considered safe for use as an adjuvant to general anesthetic in patients undergoing CEA. As demonstrated by the hemodynamic data, the MAP stability was increased following tracheal intubation in group D, as compared with group C. In addition, fewer patients were treated with nitroglycerine intraoperatively in group $\mathrm{D}$, as compared with group $\mathrm{C}$; thus suggesting that DEX was able to maintain stable circulation.

Previous studies have reported a role for CEA in stroke prevention in patients with significant carotid stenosis, including those with and without symptoms $(2,3)$. However, the effects of CEA on cognitive function remain controversial, due to mixed reports in the literature. CEA-associated cognitive dysfunction has previously been detected in $24-28 \%$ of patients following CEA $(7,8)$. Similarly, in the present study, $\sim 27 \%$ of patients exhibited a reduction in MMSE scores at
$48 \mathrm{~h}$ post-CEA, as compared with pre-surgery scores. Previous studies have suggested various possible mechanisms regarding cognitive impairment post-CEA, including cerebral ischemia during clamping, emboli from air or the surgical site, oxidative stress, and postoperative cerebral hyperperfusion $(15,16)$.

MMSE scores are commonly used as an indication of cognitive abilities, including memory, attention and language, and have been demonstrated to be a useful tool for the evaluation of cognitive dysfunction following CEA $(17,18)$. The MMSE can be conducted in $<10 \mathrm{~min}$ by following simple instructions. Various other neuropsychological scales and tests for cognitive dysfunction, including the Montreal Cognitive Assessment and Wechsler Memory Scale, are of limited use due to the complex training required (19). The present study applied the MMSE for the evaluation of cognitive dysfunction. Previous studies demonstrated that a deterioration in MMSE scores may be associated with clinically identifiable ischemic events, including subtle stroke and transient ischemic attack $(20,21)$. In both groups there was a subtle decline in MMSE scores in the early postoperative period $(\leq 48 \mathrm{~h})$, which may be attributed to asymmetry in the distribution of cerebral blood flow post-CEA $(22,23)$. In addition, general anesthesia may have various affects on cognition; previous studies have suggested that local anesthesia may be preferable to general anesthesia with regard to the effects on cognitive function $(24,25)$. In 
the present study, MMSE scores did not differ significantly between the two groups at T4 and T5; however, higher MMSE scores and a lower proportion of patients exhibiting a decline in MMSE scores were observed in group D at T2 and T3, as compared with group $\mathrm{C}$. These results suggested that DEX may promote the recovery of cognition following CEA, which is consistent with theoretical results obtained previously (26).

Previous studies have suggested that adrenergic receptors, in particular $\alpha 2$-ARs, may have an important role in promoting cognitive functions, including memory, learning and selective attention $(12,27)$. Cognition has been shown to be affected by increased stimulation at the dorsal noradrenergic bundles located in the locus ceruleus of the mesocephalon, and this is where $\alpha 2-A R s$ are located (13). Therefore, as a highly selective $\alpha 2-A R$ agonist, DEX may enhance cognitive function following CEA. Previous studies have demonstrated that DEX may exert organ-protective properties, including reducing cerebral, cardiac, intestinal and renal injury, and these effects have been shown to be abolished by atipamezole, which is an $\alpha 2-\mathrm{AR}$ antagonist (28-31). Furthermore, DEX may protect the kidney against I/R injury by inhibitory effects on injury-induced activation of the Janus kinase/signal transducer and activator of transcription signaling pathway (32). Accordingly, DEX may exert neuroprotective effects on I/R injury during CEA, and further promote the recovery of cognition in patients.

Reactive oxygen species may have a critical role in neuronal damage during CEA; the release of oxidative mediators may be partly facilitated by the injured cerebral endothelium, which may be further damaged by these mediators, leading to an impairment of cerebrovascular autoregulation (33). MDA, which is a highly reactive three-carbon dialdehyde, is a byproduct of polyunsaturated fatty acid peroxidation. In the present study, the concentration of MDA was significantly decreased in the jugular bulb of group D patients following a period of carotid clamping, as compared with group $C$; thus suggesting that DEX is able to exert antioxidative activities. However, the concentration of MDA was not significantly different between the two groups following carotid declamping, which may be due to the release of oxidative mediators from the carotid endothelium following cerebral reperfusion, which may have obscured the cerebral antioxidative effects of DEX (33). However, the decreased concentration of MDA in the DEX-treated group following carotid clamping suggest that DEX may provide an antioxidative benefit in cerebral ischemia, and this may be the basis of the improved cognitive performance associated with DEX.

In the present study, improved cognitive function and the suppression of MDA concentrations were observed to be associated with decreased protein expression levels of S100B in group D. S100B is a highly specific biochemical marker of neuronal damage, and is hypothesized to mediate nuclear factor- $\kappa \mathrm{B}$ activation and the release of proinflammatory cytokines (34). Disrupted astrocytes have been shown to release massive quantities of $\mathrm{S} 100 \mathrm{~B}$ following cerebral ischemia, which may further aggravate cerebral injury. Previous animal studies have demonstrated an important role for elevated S100B levels in cognitive decline $(35,36)$. An increased concentration of S100B may upregulate the expression of cyclooxygenase-2, which in turn may lead to the deterioration of spatial memory and learning capabilities via the receptor for advanced glycation end-products $(37,38)$. The present study detected increased protein expression levels of S100B following carotid unclamping, and this was associated with cerebral injury and a subtle decline in MMSE scores. Furthermore, decreased protein expression levels of S100B were detected in the DEX-treated group, as compared with group $\mathrm{C}$, and this appears to be associated with the neuroprotective effects of DEX. However, the underlying molecular mechanisms remain unclear.

In the present study, the serum protein expression levels of NSE were determined as an additional marker of cerebral injury; the serum protein expression levels of NSE were not significantly different between the two groups at any time point. These results may be due to the short sampling time; previous studies have reported that NSE is a promising marker of cerebral damage induced by cardiac arrest at later $(>24 \mathrm{~h})$ stages only $(39,40)$. Therefore, significant differences in the protein expression levels of NSE post-CEA between the groups may have been detected if the study duration had been increased.

Various limitations were associated with the present study, including the small number of patients that were enrolled. In addition, the cognition evaluation was conducted using a single test, whereas a combination of various cognitive function tests may have increased the objectivity of the evaluation. Furthermore, the post-CEA administration of other drugs was not standardized, which may have had knock-on effects on cognitive impairment, and the present study only investigated the effects of a single dose of DEX, such that the neuroprotective effects of DEX in larger or lower doses remain unknown. Therefore, future studies incorporating a larger sample size, and which extend the study duration to later time points post-CEA, are required.

In conclusion, the results of the present study suggested that the infusion of DEX during CEA may improve the recovery of cognitive performance post-CEA. These cognitive improvements may be due to the antioxidative effects of DEX in the ischemic cerebral circulation and associated with decreased levels of S100B. Future studies should investigate the neuroprotective potential of DEX at various doses, as well as studying the underlying molecular mechanism.

\section{Acknowledgements}

This study was funded by the National Natural Science Fund (grant no. 81171838), and the Twelfth Five-year Key Talents Project of Jiangsu Province (grant no. RC2011041).

\section{References}

1. Barnett HJ and Haines SJ: Carotid endarterectomy for asymptomatic carotid stenosis. N Engl J Med 317: 1468-1468, 1998.

2. North American Symptomatic Carotid Endarterectomy Trial Collaborators: Beneficial effect of carotid endarterectomy in symptomatic patients with high-grade carotid stenosis. N Engl J Med 325: 445-453, 1991.

3. Halliday A, Mansfield A, Marro J, Peto C, Peto R, Potter J and Thomas D; MRC Asymptomatic Carotid Surgery Trial (ACST) Collaborative Group: Prevention of disabling and fatal strokes by successful carotid endarterectomy in patients without recent neurological symptoms: Randomised controlled trial. Lancet 363: 1491-1502, 2004.

4. Berman L, Pietrzak RH and Mayes L: Neurocognitive changes after carotid revascularization: A review of the current literature. J Psychosom Res 63: 599-612, 2007. 
5. De Rango P, Caso V, Leys D, Paciaroni M, Lenti M and Cao P: The role of carotid artery stenting and carotid endarterectomy in cognitive performance: A systematic review. Stroke 39: 3116-3127, 2008

6. Irvine CD, Gardner FV, Davies AH and Lamont PM: Cognitive testing in patients undergoing carotid endarterectomy. Eur J Vasc Endovasc Surg 15: 195-204, 1998.

7. Heyer EJ, Sharma R, Rampersad A, Winfree CJ, Mack WJ, Solomon RA, Todd GJ, McCormick PC, McMurtry JG, Quest DO, et al: A controlled prospective study of neuropsychological dysfunction following carotid endarterectomy. Arch Neurol 59: 217-222, 2002

8. Heyer EJ, DeLaPaz R, Halazun HJ, Rampersad A, Sciacca R, Zurica J, Benvenisty AI, Quest DO, Todd GJ, Lavine S, et al: Neuropsychological dysfunction in the absence of structura evidence for cerebral ischemia after uncomplicated carotid endarterectomy. Neurosurgery 58: 474-480, 2006.

9. Guler G, Akin A, Tosun Z, Eskitascoglu E, Mizrak A and Boyaci A: Single-dose dexmedetomidine attenuates airway and circulatory reflexes during extubation. Acta Anaesthesiol Scand 49: 1088-1091, 2005.

10. Goyagi T, Nishikawa T, Tobe Y and Masaki Y: The combined neuroprotective effects of lidocaine and dexmedetomidine after transient forebrain ischemia in rats. Acta Anaesthesiol Scand 53: 1176-1183, 2009.

11. Sato K, Kimura T, Nishikawa T, Tobe Y and Masaki Y: Neuroprotective effects of a combination of dexmedetomidine and hypothermia after incomplete cerebral ischemia in rats. Acta Anaesthesiol Scand 54: 377-382, 2010.

12. Xu Y, YanJ,ZhouP,LiJ,GaoH,Xia Y and Wang Q: Neurotransmitter receptors and cognitive dysfunction in Alzheimer's disease and Parkinson's disease. Prog Neurobiol 97: 1-13, 2012.

13. Milstein JA, Lehmann O, Theobald DE, Dalley JW and Robbins TW: Selective depletion of cortical noradrenaline by anti-dopamine beta-hydroxylase-saporin impairs attentional function and enhances the effects of guanfacine in the rat. Psychopharmacology (Berl) 190: 51-63, 2007.

14. Sani NF, Belani LK, Sin CP, Rahman SN, Das S, Chi TZ, Makpol S and Yusof YA: Effect of the combination of gelam honey and ginger on oxidative stress and metabolic profile in streptozotocin-induced diabetic Sprague-Dawley rats. Biomed Res Int 2014: 160695, 2014

15. Wilson DA, Mocco J, D'Ambrosio AL, Komotar RJ, Zurica J, Kellner CP, Hahn DK, Connolly ES, Liu X, Imielinska C and Heyer EJ: Post-carotid endarterectomy neurocognitive decline is associated with cerebral blood flow asymmetry on post-operative magnetic resonance perfusion brain scans. Neurol Res 30 302-306, 2008

16. Wolf O, Heider P, Heinz M, Poppert H, Sander D, Greil O, Weiss W, Hanke M and Eckstein HH: Microembolic signals detected by transcranial Doppler sonography during carotid endarterectomy and correlation with serial diffusion-weighted imaging. Stroke 35: e373-e375, 2004.

17. Sabbagh M, Cummings J, Christensen D, Doody R, Farlow M, Liu L, Mackell J and Fain R: Evaluating the cognitive effects of donepezil $23 \mathrm{mg} / \mathrm{d}$ in moderate and severe Alzheimer's disease: Analysis of effects of baseline features on treatment response. BMC Geriatr 13: 56, 2013.

18. Mukonzo JK, Okwera A, Nakasujja N, Luzze H, Sebuwufu D, Ogwal-Okeng J, Waako P, Gustafsson LL and Aklillu E: Influence of efavirenz pharmacokinetics and pharmacogenetics on neuropsychological disorders in Ugandan HIV-positive patients with or without tuberculosis: A prospective cohort study. BMC Infect Dis 13: 261, 2013.

19. Arpaci AH and Bozkırli F: Comparison of sedation effectiveness of remifentanil-dexmedetomidine and remifentanil-midazolam combinations and their effects on postoperative cognitive functions in cystoscopies: A randomized clinical trial. J Res Med Sci 18: 107-114, 2013

20. Arciniegas DB, Kellermeyer GF, Bonifer NM Anderson-Salvi KM and Anderson CA: Screening for cognitive decline following single known stroke using the Mini-Mental State Examination. Neuropsychiatr Dis Treat 7: 189-196, 2011

21. Capoccia L, Sbarigia E, Rizzo A, Mansour W and Speziale F: Silent stroke and cognitive decline in asymptomatic carotid stenosis revascularization. Vascular 20: 181-187, 2012.
22. Gaunt ME, Martin PJ, Smith JL, Rimmer T, Cherryman G, Ratliff DA, Bell PR and Naylor AR: Clinical relevance of intraoperative embolization detected by transcranial Doppler ultrasonography during carotid endarterectomy: A prospective study of 100 patients. Br J Surg 81: 1435-1439, 1994.

23. Kügler CF, Funk H, Vlajic P and Platt D: The relationship between endothelin-1, event-related P300 potentials, and prognosis in cerebral arteriosclerosis. J Am Geriatr Soc 45 427-434, 1997.

24. Aleksic M, Huff W, Hoppmann B, Heckenkamp J, Pukrop R and Brunkwall J: Cognitive function remains unchanged after endarterectomy of unilateral internal carotid artery stenosis under local anaesthesia. Eur J Vasc Endovasc Surg 31: 616-621, 2006.

25. Weber CF, Friedl H, Hueppe M, Hintereder G, Schmitz-Rixen T, Zwissler B and Meininger D: Impact of general versus local anesthesia on early postoperative cognitive dysfunction following carotid endarterectomy: GALA Study Subgroup Analysis. World J Surg 33: 1526-1532, 2009

26. Chen J, Yan J and Han X: Dexmedetomidine may benefit cognitive function after laparoscopic cholecystectomy in elderly patients. Exp Ther Med 5: 489-494, 2013.

27. Galeotti N,Bartolini A and Ghelardini C: Alpha-2 agonist-induced memory impairment is mediated by the alpha-2A-adrenoceptor subtype. Behav Brain Res 153: 409-417, 2004.

28. Sanders RD, Sun P, Patel S, Li M, Maze M and Ma D: Dexmedetomidine provides cortical neuroprotection: Impact on anaesthetic-induced neuroapoptosis in the rat developing brain. Acta Anaesthesiol Scand 54: 710-716, 2010.

29. Zhang XY, Liu ZM, Wen SH, Li YS, Li Y, Yao X, Huang WQ and Liu KX: Dexmedetomidine administration before, but not after, ischemia attenuates intestinal injury induced by intestinal ischemia-reperfusion in rats. Anesthesiology 116: 1035-1046, 2012

30. Yoshitomi O, Cho S, Hara T, Shibata I, Maekawa T, Ureshino H and Sumikawa K: Direct protective effects of dexmedetomidine against myocardial ischemia-reperfusion injury in anesthetized pigs. Shock 38: 92-97, 2012.

31. Gu J, Sun P, Zhao H, Watts HR, Sanders RD, Terrando N, Xia P, Maze $\mathrm{M}$ and Ma D: Dexmedetomidine provides renoprotection against ischemia-reperfusion injury in mice. Crit Care 15: R153, 2011.

32. Si Y, Bao H, Han L, Shi H, Zhang Y, Xu L, Liu C, Wang J, Yang X, Vohra A and Ma D: Dexmedetomidine protects against renal ischemia and reperfusion injury by inhibiting the JAK/STAT signaling activation. J Transl Med 11: 141, 2013.

33. Saito H, Ogasawara K, Komoribayashi N, Kobayashi M, Inoue T, Otawara Y and Ogawa A: Concentration of malondialdehyde-modified low-density lipoprotein in the jugular bulb during carotid endarterectomy correlates with development of postoperative cognitive impairment. Neurosurgery 60: 1067-1073, 2007.

34. Mori T, Asano T and Town T: Targeting S100B in cerebral ischemia and in Alzheimer's disease. Cardiovasc Psychiatry Neurol 2010: 687067, 2010.

35. Leclerc E, Sturchler E and Vetter SW: The S100B/RAGE axis in Alzheimer's disease. Cardiovasc Psychiatry Neurol 2010: 539581,2010

36. Bell K, Shokrian D, Potenzieri C and Whitaker-Azmitia PM: Harm avoidance, anxiety, and response to novelty in the adolescent S-100beta transgenic mouse: Role of serotonin and relevance to Down syndrome. Neuropsychopharmacology 28: 1810-1816, 2003

37. Sorci G, Bianchi R, Riuzzi F, Tubaro C, Arcuri C, Giambanco I and Donato R: S100B protein, a damage-associated molecular pattern protein in the brain and heart, and beyond. Cardiovasc Psychiatry Neurol 2010: 656481, 2010.

38. Peng $M$, Wang YL, Wang FF, Chen $C$ and Wang CY: The cyclooxygenase-2 inhibitor parecoxib inhibits surgery-induced proinflammatory cytokine expression in the hippocampus in aged rats. J Surg Res 178: e1-e8, 2012.

39. Rosén H, Sunnerhagen KS, Herlitz J, Blomstrand C and Rosengren L: Serum levels of the brain-derived proteins S-100 and NSE predict long-term outcome after cardiac arrest. Resuscitation 49: 183-191, 2001.

40. Rech TH, Vieira SR, Nagel F, Brauner JS and Scalco R: Serum neuron-specific enolase as early predictor of outcome after in-hospital cardiac arrest: A cohort study. Crit Care 10: R133, 2006. 